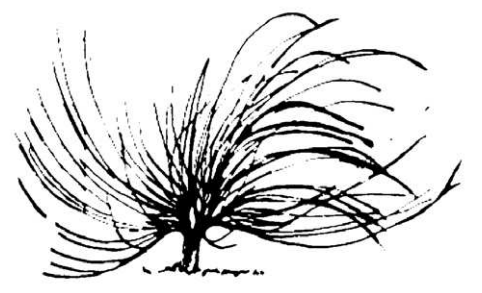

\title{
Educación en derechos humanos en universidades públicas costarricenses: posibilidades y necesidades
}

\author{
Marco Vinicio Méndez Coto ${ }^{1}$ \\ Universidad Nacional de Costa Rica \\ Heredia, Costa Rica \\ marcomendez1988@gmail.com
}

\begin{abstract}
Resumen
El artículo plantea las inconsistencias en torno a la obligación estatal por impartir y promover la educación en derechos humanos, particularmente se analiza el rol de las universidades públicas costarricenses. Dentro de los hallazgos más relevantes se destaca la escasa oferta de educación formal y no formal en la materia, así como las carencias de las organizaciones de derechos humanos en el país en cuanto a profesionalización en derechos humanos se refiere, y las expectativas de los y las estudiantes de secundaria ante un nuevo grado universitario en derechos humanos.

Como fuente principal, se utilizan los datos primarios generados por el proyecto de investigación "Necesidades sociales de formación universitaria en promoción y protección en derechos humanos" del Instituto de Estudios Latinoamericanos de la Universidad Nacional de Costa Rica (mayo 2011 - mayo 2012). Dicho proceso consistió en el análisis de las necesidades sociales de formación en derechos humanos, contando con la participación de múltiples actores vinculados a la temática.
\end{abstract}

Recibido: 18 de febrero de 2013 - Aprobado: 11 de octubre de 2013

1 Máster en Derechos Humanos y Educación para la Paz y Licenciado en Relaciones Internacionales con énfasis en Política Exterior y Diplomacia (Summa Cum Laude) de la Universidad Nacional de Costa Rica. Se desempeña como académico-investigador en la Escuela de Relaciones Internacionales y el Instituto de Estudios Latinoamericanos de la misma Universidad. 
Palabras clave: educación en derechos humanos, educación formal, educación no formal, universidades públicas costarricenses

\begin{abstract}
The article addresses the inconsistencies in the State's obligation to provide and promote human rights education, specifically analyzing the Costa Rican public universities' role. Part of the most important conclusions are the scarcity of formal and non formal educational programs in Human Rights, as well as deficiencies in Human Rights organizations, particularly related to the professionalization on Human Rights, and the expectations of high school students facing a new career on Human Rights.

Its main reference is the data provided by the research project "Social needs of university education on promotion and protection of Human Rights" by Latin American Institute of the National University of Costa Rica (May 2011 - May 2012). With the participation of multiple institutions involved in the issue, the project mentioned above analyzed social needs of university education on Human Rights.
\end{abstract}

Keywords: education in human rights, formal education, non formal education, Costa Rican public universities

\title{
Introducción
}

a educación en derechos humanos ${ }^{2}$ puede ser aprehendida como
un componente transversal del derecho a la educación, éste últi-
mo a su vez, en palabras del Comité de Derechos Económicos,

2 Para el IIDH la educación en derechos humanos consiste en: "un proceso de adquisición de determinados conocimientos, habilidades y valores necesarios para conocer, comprender, afirmar y reivindicar los propios derechos sobre la base de las normas dispuestas en los distintos instrumentos internacionales en conexión con la normativa interna. Significa que todas las personas -independientemente de su sexo, origen nacional o étnico y sus condiciones económicas, sociales o culturales- tienen la posibilidad real de recibir educación sistemática, amplia y de buena calidad que les permita: comprender sus derechos humanos y sus respectivas responsabilidades; respetar y proteger los derechos humanos de otras personas; entender la interrelación entre derechos humanos, estado de derecho y gobierno democrático; y ejercitar en su interacción diaria valores, actitudes y conductas consecuentes con los derechos humanos y los principios democráticos. Entendemos este derecho a la educación en derechos humanos como parte del derecho a la educación y como condición necesaria para el ejercicio efectivo de todos los derechos humanos (2002:15). Asimismo, el Programa Mundial para la Educación en 
Sociales y Culturales (Observación General No. 13, 1999) se constituye en un derecho "habilitante" en la medida en que es el "medio para la realización de otros derechos humanos".

El derecho a la educación por su relevancia e implicaciones necesariamente constituye una de las vías para la transformación social, la dignificación de la vida humana así como para la exigibilidad y justiciabilidad de otros derechos humanos, particularmente por ser el "epítome de los derechos humanos" (Comité DESC, Observación General No. 11), es decir, el punto de convergencia de todos los derechos, como sustento y garantía de los principios de indivisibilidad, interdependencia y universalidad (Véase Informes del Relator Especial del Derecho a la Educación 2004-2010).

En este sentido, debe destacarse la existencia de disposiciones internacionales (tanto en el sistema universal como interamericano de derechos humanos) que concretizan la obligación de los Estados por garantizar el derecho a la educación, así como la educación en derechos humanos. Si bien el objetivo no es recapitular todos los instrumentos jurídicos que sustentan estás afirmaciones, basta reseñar la Convención de Derechos del Niño (Artículos 28 y 29) así como el Protocolo de San Salvador (Artículo 13), que contienen disposiciones en la materia.

Para realizar la educación en derechos humanos, los Estados deben disponer los recursos necesarios para los procesos de capacitación, enseñanza e investigación pertinentes, a su vez, se torna imperativo ampliar la mirada más allá del ámbito educativo primario y secundario, pues la formación recibida por los docentes en el ámbito universitario no siempre tiene una mirada amplia y crítica de ellos, además de no existir programas sólidos de capacitación en derechos humanos en el seno del Ministerio de Educación Pública (Cfr. MEP 2010).

Con el objetivo de analizar la dinámica de la educación superior pública, particularmente en lo que respecta a la educación en derechos humanos, se realizaron cuatro diagnósticos que han pretendido identificar las posibilidades y necesidades de formación universitaria en

derechos humanos la concibe como: "un conjunto de actividades de educación, capacitación y difusión de información orientadas a crear una cultura universal de los derechos humanos. Una educación integral en derechos humanos no sólo proporciona conocimientos sobre los derechos humanos y los mecanismos para protegerlos, sino que, además, transmite las aptitudes necesarias para promover, defender y aplicar los derechos humanos en la vida cotidiana. La educación en derechos humanos promueve las actitudes y el comportamiento necesarios para que se respeten los derechos humanos de todos los miembros de la sociedad" (Oficina del Alto Comisionado de las Naciones Unidas para los Derechos Humanos y UNESCO, 2006). 
derechos humanos. Metodológicamente, se trabajó a partir de entrevistas semiestructuradas con dirigentes de organizaciones e instituciones ${ }^{3}$ sobre derechos humanos (participaron 21 organizaciones e instituciones) así como la aplicación de un instrumento de investigación en toda la población estudiantil de undécimo año en siete colegios costarricen$\operatorname{ses}^{4}$ (públicos y privados), para un total de 330 muestras. La investigación contó con fases documentales y con la asesoría de expertos.

Para proceder con el análisis y sistematización de los resultados, se presentará una visión general de los procesos universales recientes para la promoción de la educación en derechos humanos, con el objetivo de establecer el vínculo y la necesidad de continuar trabajando a nivel de educación superior universitaria.

Posteriormente, se destacaran los principales resultados de los diagnósticos realizados entre el año 2011 y 2012, particularmente, sobre la oferta en educación formal y no formal ${ }^{5}$ en derechos humanos en las universidades públicas costarricenses, así como las necesidades sociales y posibilidades laborales planteadas por las organizaciones e instituciones participantes sobre un grado académico en derechos humanos;

3 Colectivo Sembrando la Semilla, Defensoría de los Habitantes de la República de Costa Rica, Municipalidad de Santa Ana - Unidad de Promoción de Género, Asociación de Consultores y Asesores Internacionales, Asociación de Consultores Integración y Acción Regional, Centro de Investigación y Promoción para América Central de Derechos Humanos, Amerikano Estudios, Asamblea Legislativa de la República de Costa Rica - Unidad Técnica de Igualdad y Equidad de Género, Universidad Nacional de Costa Rica - Programa de Desarrollo Integral Comunitario Costero, Centro Internacional para los Derechos Humanos de los Migrantes, Fundación Omar Dengo, Fundación Nuestras Raíces, Universidad Nacional - Instituto de Estudios Interdisciplinarios de la Niñez y la Adolescencia, Grupo Jóvenes por los Derechos Humanos Costa Rica, Asociación Liga Internacional de Mujeres pro Paz y Libertad, sección Costa Rica, Instituto Interamericano de Derechos Humanos, Asociación Voces Nuestras, Fundación Operación GAYA Internacional, Centro Feminista de Información y Acción, Coordinadora Beso Diverso, Alianza Por Tus Derechos - Coalición de ONGs para el Seguimiento de la Convención sobre los Derechos del Niño- Costa Rica

4 Los colegios participantes en el área rural fueron el Colegio Técnico Profesional de Sabalito (Puntarenas) y el Colegio Académico Guácimo (Limón). En la zona urbana encontramos al Colegio Humanístico Costarricense y Colegio María Auxiliadora (Heredia), y Colegio Seminario, Liceo del Sur y Colegio Monterrey (San José).

5 Según Cotino (2012: 62): "la enseñanza, el aprendizaje o la educación se ha clasificado tradicionalmente en: -educación formal (educación estructurada en cuanto a objetivos educativos, duración y apoyo, realizada en el sistema escolar, desde la preescolar hasta la superior, conducente a un título o certificado). -educación no-formal (educación estructurada e intencional, complementaria o remedial realizada fuera del sistema escolar, por lo general dirigida a atender necesidades y grupos específicos y no conduce necesariamente a un título o certificado), y -educación informal (la 'escuela de la vida', no se proporciona en centros de educación y formación, no conduce necesariamente a un título o certificado y no está estructurada). 
finalmente, las expectativas e intereses en tal grado académico según los estudiantes de último nivel de secundaria. La investigación explora la pertinencia de elaborar una carrera universitaria en Promoción y Protección de derechos humanos.

\section{El Decenio de Educación en Derechos Humanos de Naciones Uni- das (1995-2004)}

La década de los noventa fue sumamente álgida. En el marco de las transiciones de poder globales, signadas por la debacle de la Unión de Repúblicas Socialistas Soviéticas y la hegemonía unipolar estadounidense que tuvo un correlato con las nuevas dimensiones, prácticas y reconfiguraciones de la seguridad internacional, las naciones del mundo debían repensar las estrategias para colocar el tema de los derechos humanos como epítome de la institucionalidad internacional, a la vez, como garantía mínima para un orden internacional respetuoso de la vida humana, tomando como referencias las experiencias vividas en las dos guerras mundiales y en los conflictos de distinto calibre acontecidos en y desde la Guerra Fría.

En ese marco contextual, a su vez marcado por el surgimiento de múltiples actores internacionales, ya no solo las naciones emergentes del colapsado circuito soviético, sino también de procesos de desintegración, principalmente europeos y africanos, y el crecimiento exponencial de organizaciones internacionales no gubernamentales con márgenes de acción globales, regionales y locales; los países, para enfrentar los nuevos desafíos debían generar procesos para que los derechos humanos llegaran a los de abajo, es decir, a las personas más excluidas y vulneradas, de tal forma que los instrumentos internacionales pudiesen ser un arma para defender la dignidad de pueblos enteros.

Si bien estas aspiraciones no necesariamente tienen una relación directamente proporcional con los eventos ocurridos durante el transcurso del Decenio, si es importante considerar que la necesidad de generar una conciencia global sobre los derechos humanos, en nuestra opinión tiene como punto de inflexión la búsqueda de garantizar condiciones para una vida digna de ser vivida, la cual aun día dista mucho de ser una realidad.

Los procesos políticos mundiales y los esfuerzos en el marco de la institucionalidad internacional de las Naciones Unidas en la promoción 
de un mundo más justo y solidario entre naciones y comunidades por medio de los procesos educativos, tienen como punto de concreción reciente la proclamación del Decenio de las Naciones Unidas para la educación en la esfera de los derechos humanos, por la Asamblea General en su resolución 49/184, de diciembre de 1994, cuyo Plan de Acción fue implementado por el Alto Comisionado de Nacionales Unidas para los Derechos Humanos (ACNUDH).

El mandato del Decenio se sustentó en instrumentos jurídicos internacionales como la Declaración Universal de Derechos Humanos (Artículo 26, inciso 2), Convención Internacional sobre la Eliminación de Todas las Formas de Discriminación Racial, Pacto Internacional de Derechos Económicos, Sociales y Culturales (PIDESC), Convención de la Eliminación de Todas las Formas de Discriminación Contra la Mujer (CEDAW) y Convención sobre los Derechos del Niño (CDN)6. $\mathrm{Su}$ marco operativo es planteado en el Plan de Acción, que establece como sus principales objetivos: la evaluación de las necesidades y la formulación de estrategias; la creación y fortalecimiento de programas para la educación en la esfera de los derechos humanos; la preparación de material didáctico; el fortalecimiento de los medios de difusión; y la difusión mundial de la Declaración Universal de Derechos Humanos (A/51/506/Add.1. Págs. 2-3).

La importancia de la proclamación del Decenio se puede visibilizar en dos vías: desde el punto de vista teórico se planteó explícitamente la promoción del enfoque de indivisibilidad e interdependencia de los derechos, sean estos económicos, sociales, culturales, civiles o políticos, deberá primar un enfoque de interconexión y vínculo, y no como ha consistido la dinámica en la esfera estrictamente estatal de amparar su cumplimiento o no en el marco de la progresividad, que implica una oposición entre derechos obligatorios y otros de alcance discrecional, siendo que en la práctica cualquier conculcación de un derecho tiene un correlato con violaciones de otros derechos (Cfr. Abramovich 2005).

Por otra parte, el Decenio da un rol protagónico a las organizaciones no gubernamentales, de base comunitaria, gobiernos locales e instituciones de investigación en la promoción de la Educación en derechos humanos (como las universidades públicas), los cuales deberían ser tomadas en consideración en la formulación de los planes

6 Adicionalmente ver (IIDH, 2002:20). 
nacionales además de contar con la posibilidad de obtener apoyo de la cooperación internacional en la implementación de sus actividades.

En el ámbito universitario, tal como plantea la Adición del SGONU al informe sobre la Aplicación del Plan de Acción, debería darse énfasis en la capacitación de capacitadores y a los encargados de diseñar planes de estudio, además de solicitarse a las universidades y programas e instituciones de capacitación, el diseño de planes de estudio en materia de derechos humanos y el desarrollo de investigaciones sobre derechos humanos y educación en la esfera de los derechos humanos.

El Plan de Acción del Decenio contó en su mandato con tres evaluaciones, la primera de ellas en 1995, consistió en el diagnóstico preliminar de la situación mundial en la educación en la esfera de los derechos humanos, asimismo, en el año 2000 el ACNUDH desarrolló una evaluación de medio período y en 2004 la evaluación final del Decenio.

En el informe A/55/360 de la ACNUDH para evaluar los alcances del Decenio a la mitad del mandato, se planteó que muchas instituciones de educación en derechos humanos no han participado en el examen, de hecho, la participación de los Estados ha sido pobre, incluido Costa Rica, quien no remitió su evaluación en dicho examen.

En términos generales se estimó que muy pocos Estados desarrollaron los planes nacionales, y que las estrategias de aplicación de la educación en derechos humanos se implementaban por la vía de revisión de textos escolares con el fin de eliminar contenidos discriminatorio u opuestos a los propósitos de la DUDH, además de integrar la educación en derechos humanos a las distintas materias, y no limitándose a una sola materia.

Sobre las tendencias transregionales, muchas de las actividades en educación en derechos humanos se realizaron independientemente de los planes nacionales, siendo considerados como esfuerzos aislados e inconexos que pueden incidir negativamente a largo plazo; en cuanto a la implementación curricular, únicamente los centros especializados en derechos humanos estudian los derechos humanos como materia separada; en términos de la evaluación general, se destaca la brecha entre la retórica gubernamental y los recursos asignados en el marco del Decenio.

Para el año 2004 se presenta el informe sobre los logros y fallos en el Decenio por parte del ACNUDH (E/CN.4/2004/93). Al igual que en los informes previos, la participación por parte de los Estados fue baja, contando en éste Informe el aporte de tan solo 27 Estados, de los 
cuales Costa Rica continuo ausente. Si bien los logros son genéricos, se reafirmó que el Decenio puso la educación en derechos humanos en la palestra mundial, algunos Estados aprovecharon la ocasión para implementar planes nacionales, comités de coordinación y revisión de contenidos curriculares. Siendo destacable el hecho de la creación de institutos de investigación y licenciaturas en derechos humanos en el marco del Decenio.

\section{El Programa Mundial para la Educación en Derechos Humanos (2005-2014)}

Al finalizar el Decenio de Educación en Derechos Humanos se pretendía relanzar un segundo Decenio con el objetivo de atender los fallos y asuntos pendientes del primero, y con la consigna de que dichas estrategias educativas deben implementarse a largo plazo. En 2004, como continuidad del Decenio se aprueba el Programa Mundial para la Educación en Derechos Humanos (PMEDH) (2005-2014), que será abordado en distintas fases, siendo la primera de ellas la educación primaria y secundaria (Cfr. E/CN.4/2005/98).

\section{a. Plan de acción PMEDH. Primera etapa 2005-2007}

El Plan de Acción aprobado para la primera etapa consistía en cuatro fases para diagnosticar, planificar, implementar y evaluar estrategias o planes nacionales de educación en derechos humanos en la educación primaria y secundaria, teniendo como ente rector los mismos ministerios de educación de cada país (OACNUDH y UNESCO, 2006). En principio el Plan se implementaría hasta el año 2007, sin embargo su mandato fue ampliado hasta el 2009 cuya evaluación final se da en 2010, participando Costa Rica en la misma.

En el caso costarricense fue destacada la creación de la Dirección de Promoción y Protección de los Derechos, "responsable de promover una cultura institucional sustentada en la promoción y protección de los derechos humanos y, específicamente, en el cumplimiento y defensa de los derechos" (MEP 2010). Adicionalmente se reconoció tácitamente la inexistencia de un Plan Nacional en Derechos Humanos, pues lo que existe para el abordaje -tal como señala el MEP- es "un único eje trasversal que permea todos los planes de estudio de la educación pública costarricense, denominado Valores... operacionalizado a través de 4 temas 
relacionados que son: Cultura Ambiental para el Desarrollo Sostenible, Educación Integral de la Sexualidad, Educación para la Salud y Vivencia de los Derechos Humanos para la Democracia y la Paz" (2010).

\section{b. Plan de acción PMEDH. Segunda etapa 2010-2014}

En el año 2009 la Oficina del ACNUDH elaboró una consulta mundial sobre el objeto de la segunda etapa del PMEDH para el período 2010-2014. Tras la consulta global se obtuvo que "en 15 respuestas se identificó la universidad o la enseñanza terciaria como sector destinatario para la siguiente etapa, haciendo una proyección lógica a partir de la focalización actual en la enseñanza primaria y secundaria" (A/ HRC/12/36, Par. 75). Debe señalarse que múltiples gobiernos plantearon continuar trabajando en la enseñanza primaria y secundaria, así como otros ámbitos, tal fue el caso de Costa Rica al pronunciarse favorable por centrar la atención en la educación en los derechos sexuales y reproductivos de los jóvenes (A/HRC/12/36, Parágrafo. 10).

Finalmente se decide centrar la segunda etapa (2010-2014) en la Educación en derechos humanos en la enseñanza superior y en los Programas de formación en derechos humanos para docentes y educadores, funcionarios públicos, fuerzas del orden y personal militar. Según el nuevo Plan de Acción "la educación en derechos humanos en la enseñanza superior debe concebirse como un proceso que incluye: a) 'Los derechos humanos por conducto de la educación', a fin de asegurar que todos los componentes y procesos del aprendizaje, incluidos los planes de estudio, los materiales, los métodos y la formación misma conduzcan al aprendizaje de los derechos humanos; b) 'Los derechos humanos en la educación', a fin de asegurar que se respeten los derechos humanos de todos los interesados, y el ejercicio de esos derechos, en el sistema de enseñanza superior (A/HRC/15/28, Par. 22).

El Plan de Acción requerirá de los Estados, bajo el respeto de la autonomía universitaria, la propensión por elaborar programas académicos inter y multidisciplinarios, tanto de grado como de posgrado en derechos humanos, así como armonizar las políticas educativas y conexas para que integre la enseñanza superior en los planes relacionados con la Educación en derechos humanos.

Durante el año 2012 se realizó la evaluación de medio período (A/ HRC/21/20), manifestándose la ausencia de Costa Rica, adicionalmente, los resultados reseñados continúan siendo básicos a nivel mundial, 
pues se anota como logros la mera existencia de materias en grados académicos sin la presencia de planes o propuestas programáticas. La segunda fase del PMEDH resulta fundamental tomando en cuenta la búsqueda a nivel mundial de instaurar y consolidar la enseñanza de derechos humanos en la educación superior, situación en Costa Rica a la cual el presente trabajo presta atención particular.

\section{La educación formal en derechos humanos en universidades públi- cas de Costa Rica ${ }^{7}$ : situación actual}

A partir del marco brindado por el Decenio y el Programa Mundial de Educación en Derechos Humanos se torna necesario conocer la situación actual del país en el prisma de la Educación en derechos humanos, particularmente centrados en la educación terciaria que se corresponde con la fase actual del PMEDH. En este sentido, se ha logrado identificar una escasez de programas de educación formal en derechos humanos en las universidades públicas costarricenses; pues existen programas académicos que realizan aproximaciones tangenciales a su objeto de estudio, sin embargo ninguno de ellos lo incorpora explícitamente, salvo los programas a nivel de posgrado que a continuación se identifican:

7 En este caso se incluyen la Universidad para la Paz por tener un mandato universal y sede en Costa Rica, así como el Instituto Interamericano de derechos humanos por tener un mandato regional y sede también en Costa Rica. 
Tabla 1. Programas de posgrado en Derechos Humanos por universidad. 2011.

\begin{tabular}{|c|c|}
\hline Universidad & Programa académico \\
\hline Universidad Nacional (UNA) & $\begin{array}{l}\text { 1. Maestría en Derechos Humanos y Educación } \\
\text { para la Paz }\end{array}$ \\
\hline $\begin{array}{l}\text { Universidad de Costa Rica } \\
\text { (UCR) }\end{array}$ & $\begin{array}{l}\text { 2. Maestría en Derecho Comunitario y } \\
\text { Derechos Humanos } \\
\text { 3. Maestría en Derechos de la Niñez y la } \\
\text { Adolescencia }\end{array}$ \\
\hline $\begin{array}{c}\text { Universidad Estatal a Distancia } \\
\text { (UNED) }\end{array}$ & 4. Maestría en Derechos Humanos \\
\hline $\begin{array}{c}\text { Universidad para la Paz } \\
\text { (UPAZ) }\end{array}$ & $\begin{array}{l}\text { 5. Maestría en Derecho Internacional y } \\
\text { Derechos Humanos } \\
\text { 6. Maestría en Estudios de Paz Internacional } \\
\text { 7. Maestría en Educación para la Paz }\end{array}$ \\
\hline
\end{tabular}

Fuente: Elaboración propia (2011).

Las opciones de posgrados profesionales y académicos son fundamentales en el proceso de profesionalización en derechos humanos, más importante aún, tomando en consideración el contexto mundial y los sistemas de protección de derechos humanos, particularmente el sistema interamericano por ser el área en el cual nuestro país se encuentra inmerso.

A pesar de haber un discurso en Costa Rica referido con la promoción y protección de los derechos humanos, han sido insuficientes los esfuerzos en cuanto a la formación de profesionales, relegando en la mayoría de las ocasiones el tema a los y las juristas, a pesar de ser el área de estudio por su propia naturaleza de carácter multi e interdisciplinario.

Un elemento que se destaca es el acceso a la educación en derechos humanos, pues la existencia de programas de posgrado es restrictiva par amplios grupos de personas que por condiciones sociales y económicas no tienen la posibilidad de sufragar la inversión, o la misma posibilidad de acceder a becas, generalmente concentradas en los niveles de grado y pregrado. Este proceso de exclusión a nivel nacional se evidencia en las estadísticas del Programa Estado de la Nación, quien señala que únicamente el $25.8 \%$ de la población de 18 a 24 años asiste a la enseñanza superior, sea en el ámbito privado o público (2011, p.177). 
Tabla 2. Inversión, duración, creditaje y requisitos de ingreso a los programas de Posgrado en Derechos Humanos impartidos en Costa Rica. 2011

\begin{tabular}{|c|c|c|c|c|c|}
\hline Programa & $\begin{array}{c}\text { Inversión } \\
\text { (USD) }\end{array}$ & Duración & Idiomas & Creditaje & $\begin{array}{c}\text { Prom. de } \\
\text { ingreso }\end{array}$ \\
\hline $\begin{array}{c}\text { Maestría en Derechos } \\
\text { Humanos y Educación } \\
\text { para la Paz }\end{array}$ & $2.000,00$ & 20 meses & $\begin{array}{c}\text { Inglés o } \\
\text { portugués }\end{array}$ & 60 & $8,50+$ \\
\hline $\begin{array}{c}\text { Maestría en Derechos } \\
\text { Humanos de la Niñez y la } \\
\text { Adolescencia }\end{array}$ & $4.920,00$ & 24 meses & $\begin{array}{c}\text { Inglés o } \\
\text { portugués }\end{array}$ & 61 & $8,00+$ \\
\hline $\begin{array}{c}\text { Maestría en Derechos } \\
\text { Humanos }\end{array}$ & $3.000,00$ & 16 meses & $\begin{array}{c}\text { Dominio de } \\
\text { otro idioma }\end{array}$ & 62 & N.A. \\
\hline $\begin{array}{c}\text { Maestría en Derecho } \\
\text { Comunitario y Derechos } \\
\text { Humanos }\end{array}$ & $4.550,00$ & 24 meses & N.A. & 65 & $8,50+$ \\
\hline $\begin{array}{c}\text { Maestría en Derecho } \\
\text { Internacional y Derechos } \\
\text { Humanos }\end{array}$ & $26.352,00$ & 11 meses & Inglés & 40 & N.A. \\
\hline $\begin{array}{c}\text { Maestría en Estudios de } \\
\text { Paz Internacional }\end{array}$ & $26.352,00$ & 11 meses & Inglés & 40 & N.A. \\
\hline $\begin{array}{c}\text { Maestría en Educación } \\
\text { para la Paz }\end{array}$ & $26.352,00$ & 11 meses & Inglés & 40 & N.A. \\
\hline $\begin{array}{c}\text { Curso Interdisciplinario } \\
\text { en Derechos Humanos* }\end{array}$ & $1.250,00$ & 12 días & N.A. & N.A. & N.A. \\
\hline
\end{tabular}

*No es un programa de posgrado, pero es un programa de formación continua en derechos humanos auspiciado por el IIDH.

Fuente: Elaboración propia a partir de datos en los sitios oficiales de los programas académicos. 2011.

De la Tabla No. 2 se puede analizar las diferencias en cuanto al monto de inversión requerido. El programa ofrecido por la Universidad Nacional es en un 33,33\% más asequible en comparación con el segundo programa con costes de inversión menores (UNED), en relación con los programas ofrecidos por la Universidad de Costa Rica es aproximadamente un $60 \%$ más asequible, y en comparación con los programas de la Universidad para la Paz, apenas representa el 7.5\% de la inversión total.

Como verá él y la lector/a, se ha incorporado el Curso Interdisciplinario en Derechos Humanos desarrollado anualmente por el IIDH, con los datos ofrecidos en su edición 2011. Comparativamente dicho curso representa el 62,5\% de la inversión total de la Maestría profesional ofrecida por la Universidad Nacional, a pesar de que dicho esfuerzo académico (del IIDH) no tiene un valor en términos de creditaje universitario ni es conducente a titulación universitaria. 
En relación con la duración de los programas, se encuentran desde los 11 meses que consisten en las maestrías de la Universidad para la Paz hasta los 2 años que es el promedio en la Universidad de Costa Rica. El programa de la Universidad Nacional se ubica en un punto medio entre el Programa de la UNED (16 meses) y la UCR (24 meses) al durar 20 meses (cinco trimestres).

Con respecto a los perfiles de ingreso, los programas de la UNA, UNED Y UPAZ son de carácter multidisciplinario; en el caso de la Maestría en Derecho Comunitario y Derechos Humanos es requisito ser operador jurídico con nivel mínimo de licenciatura, y para la Maestría en Derechos Humanos de la Niñez y la Adolescencia, se solicita "preferentemente licenciatura en Trabajo Social, Psicología, Derecho, Educación, Orientación o en otras áreas afines que determine la Dirección del Programa" (CONARE-OPES, 2007:10).

A partir del "Diagnóstico de la oferta de educación superior en derechos humanos en Costa Rica" elaborado en 2011 no se encontró ningún programa de pregrado o grado cuyo objeto de estudio sea explícitamente derechos humanos, como sí existen en otros países (México por ejemplo). Si bien el Ministerio de Educación Pública (2010) señala que en la formación previa del personal docente se cuenta con formación en el tema de derechos humanos, debe decirse que generalmente es un abordaje transversal en los currículos universitarios, o en cursos particulares de las mallas curriculares (Cfr. Meoño 2008), lo que no quiere decir que existan grados en Derechos Humanos.

\section{La educación no formal en derechos humanos en universidades pú- blicas costarricenses}

Uno de los componentes fundamentales en la educación en derechos humanos es la educación no formal que consiste en procesos de capacitación a la sociedad en general, siendo en ese sentido más democrático al permitir socializar la investigación universitaria y científica a un público meta más amplio, así como incidir en los niveles más locales de acción (base comunitaria).

Durante el año 2011 se realizó el diagnóstico "Oferta programas de educación permanente en derechos humanos en universidades públicas costarricenses", a saber el Instituto Tecnológico de Costa Rica (ITCR), la Universidad Estatal a Distancia (UNED), la Universidad 
Nacional (UNA), la Universidad de Costa Rica (UCR) y la Universidad Técnica Nacional (UTN). En todas las instituciones se realizó un proceso de triangulación que incluyó comunicaciones personales y visitas a los y las responsables para verificar la información y obtener guías o referencias que permitieran más validez científica.

A partir de la investigación se encontró que el Instituto Tecnológico de Costa Rica (ITCR) no cuenta con ningún programa de educación no formal relacionado con derechos humanos. Se consultó a la Sra. Ileana León Boza de la Dirección de Proyectos, y señaló que no tienen ninguno [programa y/o curso de educación no formal] relacionado con Derechos Humanos (2011).

En el caso de la Universidad Estatal a Distancia contaba con cinco programas de extensión, sin embargo a partir de consultas personales se encontró que no existían espacios destinados explícitamente a derechos humanos. Por ejemplo, se consultó a la M.Sc. Rose Mary Munguía Romero, encargada del proyecto de "Niñez y Adolescencia" de la Dirección de Extensión, quien señaló que la UNED no cuenta explícitamente con cursos relacionados con Derechos Humanos, aunque en alguna medida éstos se incorporan transversalmente. En el caso del Programa de Posgrado en Derechos Humanos del mismo recinto, se manifestó en el momento de la consulta, que únicamente se impartía la malla curricular del posgrado.

Por lo anterior se puede concluir que la UNED no cuenta con programas de educación no formal relacionada con Derechos Humanos, aunque de forma transversal se han desarrollado talleres y cuentan con un posgrado en Derechos Humanos; no hay una oferta para el público en general ni para los graduados/as de la UNED en particular.

Para diagnosticar la situación de los programas de educación no formal en la Universidad Nacional de Costa Rica se contó con el apoyo del M.Sc. Ángel Blas, Director de la Oficina de Educación Permanente. Según conversaciones sostenidas con el M.Sc. Blas, la existencia de programas de educación no formal en la Universidad Nacional ha sido incipiente, y uno de los problemas que se logró detectar es el sub-registro de las actividades académicas de extensión y actualización docente, pues muchos proyectos de Investigación y Extensión tienden a incluir dentro de sus resultados: talleres, cursos, actualizaciones profesionales, actividades abiertas a la comunidad nacional, no obstante, estás no cuentan con un registros sistemático y tampoco cuenta con una programación periódica, sino que tienden a ser coyunturales. 
Entre los hallazgos más relevantes, se contó con la colaboración del Director de Instituto del Niño (INEINA) Lic. Rodolfo Vicente Salazar, quien señaló que el INEINA si tiene cursos relacionados con derechos humanos, particularmente relacionados con los derechos del niño así como de la Convención sobre Derechos del Niño y Legislación de Niñez y Adolescencia (2011). Asimismo, en el Instituto de Estudios Latinoamericanos (IDELA) se cuenta con la Cátedra Alessandro Baratta que ha desarrollado cursos de aprovechamiento como "Criminología, política criminal y derechos humanos". Sin embargo, debe destacarse que no se cuenta con este tipo de información sistematizada a nivel universitario.

En el caso de la Universidad de Costa Rica, constituyéndose ésta en el recinto más grande y con mayor cantidad de programas de educación formal y no formal, se contó con el apoyo de la Lic. Marianela González Zúñiga de la Vicerrectoría de Acción Social. En términos generales se señala la existencia de aproximadamente 200 programas de no formal en el recinto universitario, no obstante, después de consultas en los programas más cercanos al objeto de la investigación se evidenció la inexistencia de programas de educación no formal en derechos humanos. Finalmente, en la Universidad Técnica Nacional, se encontraron tres programas de educación no formal, ninguno de ellos tiene relacionado con derechos humanos.

En términos generales se puede señalar que las universidades públicas costarricenses no poseen una oferta sistemática en educación no formal relacionada con derechos humanos. Si bien se cuentan con iniciativas que atienden a poblaciones específicas y en condiciones particulares de vulnerabilidad, no se encontró un programa sistemático que permita a la comunidad universitaria y nacional capacitarse, informase y debatir sobre temas de derechos humanos.

Sobre esta misma carencia, en el caso del Ministerio de Educación Pública se señala haber "avanzado en el tema de capacitación, a través de la reestructuración del Instituto de Desarrollo Profesional Docente Uladislao Gámez..." sin embargo "Según información del Departamento de Planes y Programas del Instituto, no existen planes particulares sobre la temática, pero dentro de algunas capacitaciones se incluyen contenidos relacionados a los Derechos Humanos, de forma trasversal..." (2010). 


\section{Las necesidades sociales y posibilidades laborales de un pro- grama académico en promoción y protección de derechos humanos según las organizaciones e instituciones consultadas}

Conociendo la inexistencia de programas sistemáticos de capacitación en derechos humanos en las universidades públicas costarricenses, así como la limitada oferta en educación formal, se realizó un diagnóstico con veintiuna organizaciones e instituciones que trabajan en derechos humanos sobre las necesidades sociales de formación universitaria en la materia, así como las posibilidades laborales que podrían tener estos nuevos profesionales.

Primeramente se preguntó a las instituciones si cuentan con personal graduado en derechos humanos, obteniéndose que diez respondieron "no", es decir, el 48\% de las organizaciones participantes a pesar de trabajar directamente la temática de derechos humanos no tienen personal graduado en el área. Tres organizaciones plantearon que a pesar de no tener graduados, sí tienen personal con capacitaciones en derechos humanos, y ello les permite su abordaje teórico y conceptual; estas organizaciones representan el 14\% de la muestra. Finalmente, el $38 \%$ señaló que "sí" tienen profesionales graduados en derechos humanos.

Tomando en consideración que la mayoría de las organizaciones no posee profesionales en el área, se consultó por las principales dificultades en términos teóricos y profesionales del abordaje de derechos humanos. Destacándose en términos generales como una de las principales dificultades los prejuicios sociales sobre "derechos humanos" así como el desconocimiento de la población de los mismos. Estos se traducen en una naturalización de discriminación, reproducción de prejuicios y desinterés por la exigibilidad y justiciabilidad de derechos humanos frente a sistemas jurídicos ajenos a la realidad de los más excluidos. Este desconocimiento de la población sobre derechos humanos no se debería únicamente a la carencia de programas nacionales de capacitación, sino que, como alegan las organizaciones, las mismas comunidades pueden sentirse ajenas a la temática por lo abstracto de los planteamientos y la carencia de material amigable sobre derechos humanos.

Dentro de las dificultades profesionales se plantea la falta de capacitación de los agentes públicos para la defensa de derechos humanos, así como la dificultad para acceder a espacios de formación, 
tomando en cuenta la limitada oferta existente y el esfuerzo económico que implica para organizaciones locales o profesionales liberales.

Se consultó a las organizaciones participantes sobre las estrategias de actualización o capacitación con que cuentan, obteniéndose que el $52 \%$ manifestaron la existencia de procesos, no obstante la mayoría de las veces son coyunturales (por motivaciones personales o por oportunidades), y no necesariamente responden a un proceso global de la organización que se relacione con políticas de actualización y de acercamiento profesional a la temática, tal como se expresa en la Figura No. 1.

Figura 1. Procesos de capacitación o actualización que disponen las organizaciones participantes. 2012.

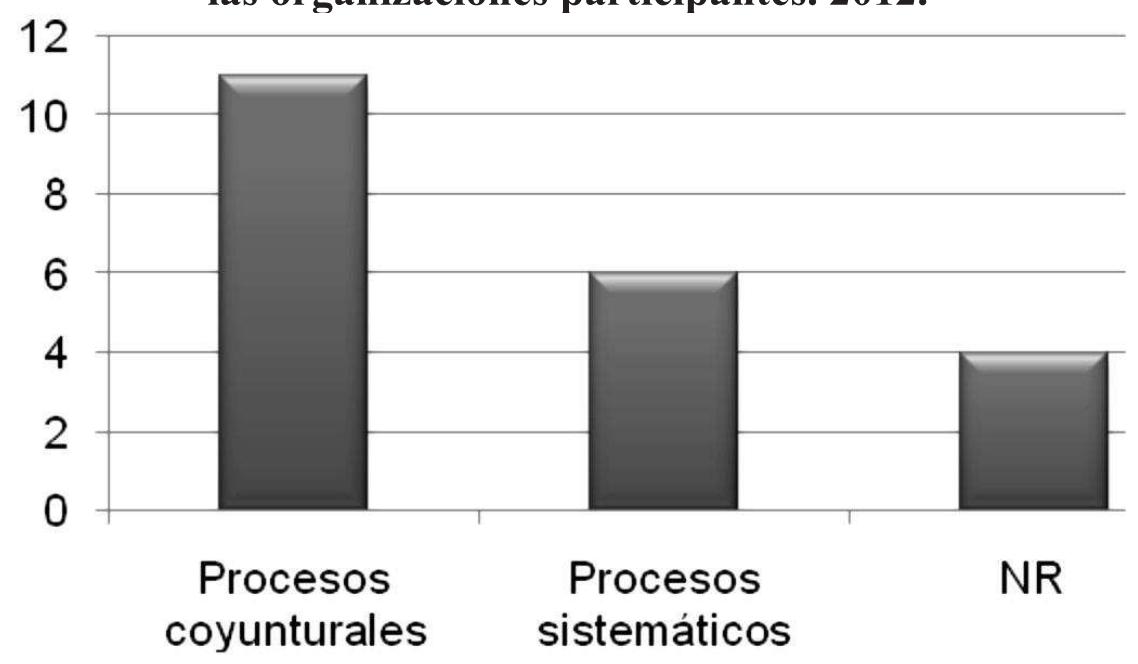

Fuente: Elaboración propia a partir de datos obtenidos en el proceso de consulta. 2012.

Del total de organizaciones, solamente el $29 \%$ señaló poseer procesos de capacitación incorporados como políticas institucionales, para lo cual cuentan con convenios o redes interinstitucionales que permiten que el personal pueda acceder a ellas. Se destaca el hecho de que para tres organizaciones estas capacitaciones son requisitos de contratación, tal como sugiere el Plan de Acción de la Segunda Etapa del PMEDH.

A partir de los datos aportados se evidencia la ausencia de titulación formal en derechos humanos en la mayoría de las organizaciones e instituciones, junto a procesos de capacitación coyunturales o motivados por intereses particulares, lo que podría indicar que las organizaciones no siempre cuentan con perspectivas teóricas sólidas, o que las posibilidades 
reales de actualización no son asequibles para la mayoría, siendo esto una oportunidad para las universidades públicas costarricenses.

$\mathrm{Al}$ preguntar a las organizaciones sobre las posibilidades reales de incorporar al graduado laboralmente, éstas indicaron que es una posibilidad real en el $29 \%$ de las organizaciones, el $9 \%$ de las organizaciones señaló que esta posibilidad se encuentra limitada o sujeta al aumento de recursos públicos por parte del Estado para abrir plazas, pero de ser así consideran que sí es una posibilidad, además otro $10 \%$ de las organizaciones indicó que esto estaría sujeto a los recursos generados por concepto de cooperación internacional a través de proyectos, tal como señala la Figura 2.

Figura 2. Posibilidades reales de incorporar laboralmente al profesional graduado en derechos humanos. 2012.

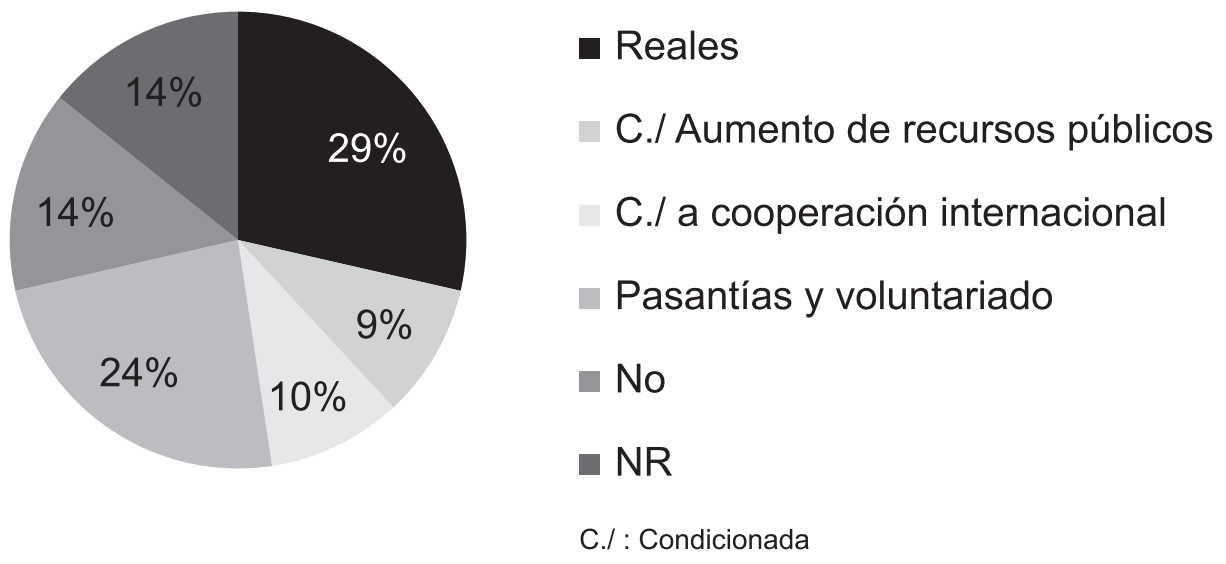

Fuente: Elaboración propia a partir de datos obtenidos en el proceso de consulta. 2012.

Un segundo grupo (24\%) consistió en aquellas organizaciones que señalaron posible recibir a estos graduados en pasantías o prácticas profesionales, esto se explica porque algunas organizaciones funcionan a partir del voluntariado y no necesariamente a partir de contrataciones laborales directas, y también se argumentó que dentro de los procesos culturales de las organizaciones, muchas veces la pasantía es un paso previo a la contratación. Existe un 14\% que señaló que no es posible incorporar a estos profesionales y otro $14 \%$ no respondió.

Finalmente al preguntar a las organizaciones si tomando en consideración su incidencia y el trabajo que realizan ¿es una necesidad social contar con un grado académico en derechos humanos? 
respondiendo el $81 \%$ de las organizaciones que sí es una necesidad social y un aporte a la sociedad costarricense muy importante, el 14\% señaló que no lo consideran necesario, particularmente se comentó el tema de las posibilidades laborales, y el 5\% restante no respondió, tal como demuestra la Figura 3.

Figura 3. Necesidades sociales de la creación de un grado académico en derechos humanos. 2012.

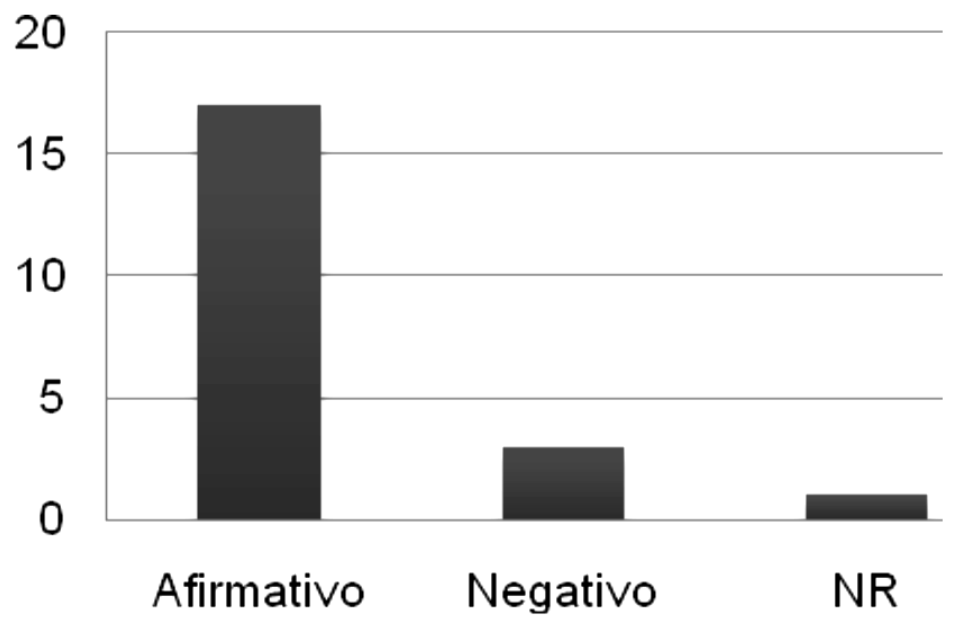

Fuente: Elaboración propia a partir de datos obtenidos en el proceso de consulta. 2012.

A partir de los datos ofrecidos de la consulta con las organizaciones, se puede comprender la importancia de fortalecer la educación universitaria en derechos humanos en Costa Rica, particularmente con la oportunidad que abre el Programa Mundial de Educación en Derechos Humanos, cuya segunda fase insta a los gobiernos y a las universidades a fomentar, crear y promover la educación, investigación y capacitación en derechos humanos.

Intereses y expectativas de población estudiantil sobre la creación de una carrera universitaria en promoción y protección de derechos humanos

Considerando los resultados de la consulta a las organizaciones e instituciones sobre las necesidades sociales, se desarrolló en el año 2012 un Diagnóstico con estudiantes de último año de secundaria, siendo el objetivo conocer sus intereses y expectativas en relación con la 
eventual creación de un grado universitario en Promoción y protección de derechos humanos. Con una muestra significativa de 330estudiantes, se determinó que aun cuando no existe un grado académico en el país en el área de estudio propuesta, los y las estudiantes mostraron una recepción inicial positiva a la misma.

El Diagnóstico utilizó una muestra no probabilística (Hernández, 2006, p. 241), particularmente se incluyeron en la muestra estudiantes provenientes de colegios rurales y urbanos por una parte, y públicos, privados y semiprivados por otra parte ${ }^{8}$. Adicionalmente se requería que los y las estudiantes estuviesen cursando el último nivel de secundaria (potenciales estudiantes universitarios de 2013).

En el estudio participaron 330 estudiantes de los cuales 188 eran mujeres y 141 eran hombres. Desagregando los datos se destaca que del total de estudiantes, 259 correspondían a zonas urbanas y 71 a zonas rurales, siendo sus rangos etarios principalmente los 16 años (104 casos) y 17 años (142 casos).

Se consultó a los y las estudiantes si tenían interés en cursar una carrera en Promoción y protección de derechos humanos, resultado afirmativo en el $27 \%$ de los casos, $4 \%$ planteó su interés de cursarla como "segunda carrera", y el 11\% indicó que consideran posible cursarla, sin embargo esto quedaría supeditado a la obtención de mejor y mayor información y claridad en el ámbito laboral. Mientras que el $57 \%$ indicó no tener interés en cursar una carrera en este sentido, como demuestra la Figura 4.

8 Para la selección de los centros educativos se utilizaron datos brindados por el Ministerio de Educación Pública. 
Figura 4. Balance de expectativas de estudiantes de secundaria de cursar Promoción y Protección en Derechos Humanos

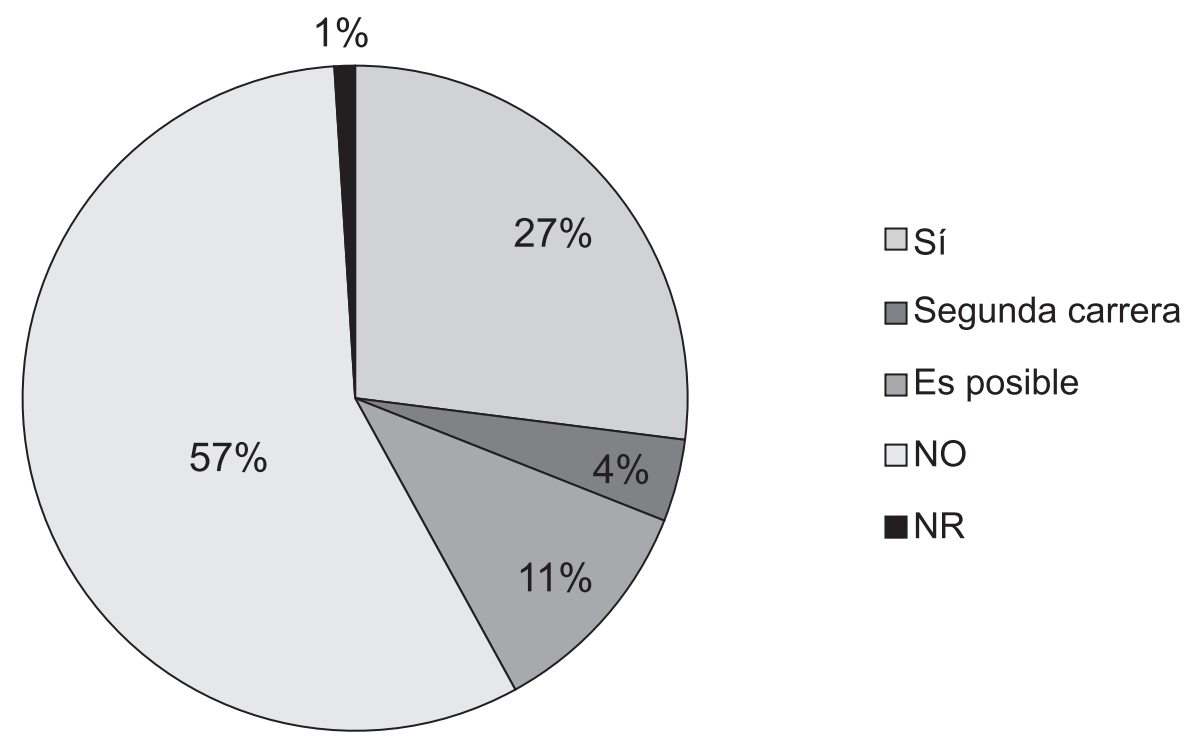

Elaboración propia a partir de consulta con estudiantes. 2012.

De los datos aportados por la investigación se pueden extraer dos conclusiones, la primera es que de la totalidad de la muestra existe de una importante población estudiantil con distintos grados de interés en la nueva carrera (42\%), siendo que de este total un $27 \%$ había expresado su interés real/prioritario. Una segunda conclusión preliminar destacaría el hecho de que aun cuando el 57\% indicó no tener interés, debe señalarse que los y las estudiantes no saben qué perfil profesional tendría esta nueva carrera ni el nicho laboral que nutriría, siendo estos factores negativos por sí mismos.

Delimitando las preferencias entre quienes manifestaron su interés en ingresar a la nueva carrera por sexo, se observa una mayor preferencia entre las mujeres $(46,6 \%)$ que de los hombres $(36,1 \%)$, asimismo, entre quienes demostraron su interés como segunda carrera las mujeres tienen más determinación en ingresar $(31,4 \%)$ que los hombres $(21,3 \%)$.

En el instrumento de investigación se incluyeron dos preguntas abiertas que tenían como objetivo conocer las expectativas e intereses de los estudiantes en esta nueva carrera, particularmente en lo que respecta a las poblaciones que la nueva profesión debería abordar como prioritaria, así como los conocimientos y habilidades que debería tener un graduado en Promoción y Protección en Derechos Humanos. 
Metodológicamente se solicitó a los y las estudiantes señalar, en cada una de las preguntas, tres grupos de población de interés, y tres habilidades o conocimientos que propicie la nueva carrera. En ese sentido, se contó con un universo de 990 respuestas para cada pregunta, considerando que son tres para cada una de las 330 muestras. Estas respuestas fuero analizadas a partir de grandes categorías creadas por el investigador responsable, que abarcan un conjunto de ideas sobre un mismo tema (grupo social, habilidad o conocimiento).

Sobre los grupos de población seleccionados se obtuvo una mayor identificaron con grupos con características específicas de vulnerabilidad, destacándose en orden de relevancia los niños, niñas y adolescentes (Niñez 177 casos y Adolescentes 172 casos). Posteriormente encontramos a los y las adultos/as mayores (137 casos), personas adultas -desde 18 hasta 65 años- (76 casos) así como los grupos indígenas (63 casos).

En lo que respecta a las habilidades o conocimientos propuestos por los y las estudiantes, estos fueron separados en tres grandes grupos, uno primero referido a contenidos teóricos o áreas de conocimiento científico (G1); el segundo grupo hace referencia a destrezas para el ejercicio profesional de los y las estudiantes y graduadas/os (G2), y un tercero sobre las habilidades o competencias sociales que se deben exigir de un profesional en derechos humanos (G3).

Con respecto al primer grupo (G1) se dio prioridad al tema del Derecho (110 casos), siendo este fundamental como pilar del currículo académico. Seguidamente se creó una categoría intitulada "derechos humanos" (92 casos) en la cual se contemplaron sus teorías, categorías, conceptos y la investigación (en derechos humanos), finalmente la Protección de los derechos humanos (76 casos) relacionado con la capacidad de defenderlos, siendo los tres más significativos.

En relación con el segundo grupo de categorías (G2) encontramos que las destrezas que requiere este nuevo profesional empiezan por una buena expresión oral, escrita y de comunicación efectiva (69 casos), los y las estudiantes apuntan que es prioritario para Derechos Humanos profesionales capacitados para poder debatir y enfrentarse públicamente con distintos actores, esto es fundamental pues la defensa y promoción de derechos humanos debe ser activa. Añaden como segunda destreza las herramientas para capacitar y enseñar a personas o grupos (30 casos), resultado interesante comparando los datos brindados por las Organizaciones de Derechos Humanos en cuanto a la necesidad de actualización permanente en el área. 
Finalmente, en el tercer grupo de categorías (G3) se reconoce la creencia en que este nuevo profesional deberá ser una persona con disposición para relacionarse con los demás, por ello se destaca como prioritario el interés de ayudar y ser solidarios/as (50 casos); tratar bien a las personas (45 casos) y promover la igualdad, equidad social y la no discriminación (39 casos).

\section{Consideraciones finales}

A lo largo del artículo se ha reseñado cómo en el marco del Programa Mundial para la Educación en Derechos Humanos, concretamente en su segunda fase destinada a la educación superior universitaria, se alienta a los Estados e instituciones de enseñanza a la creación de programas académicos en derechos humanos. En ese marco se desarrollaron diagnósticos sobre la oferta formal y permanente en las universidades públicas costarricense, destacándose la existencia de pocos programas formales de educación en derechos humanos conducente a titulación universitaria, únicamente en nivel de posgrado.

Las universidades públicas costarricenses no poseen una oferta formal en educación permanente o continua relacionada con derechos humanos. Si bien existen iniciativas que atienden a poblaciones específicas y en condiciones particulares de vulnerabilidad, no se encontraron programas sistemáticos que permitan a la comunidad universitaria y nacional capacitarse, informase y debatir sobre temas de derechos humanos, misma situación que ocurre al interior del Ministerio de Educación Pública, cuando de la capacitación docente se trata.

Es particularmente revelador que, a pesar de ser Costa Rica un país que proclama la defensa y tutela de derechos humanos, no exista una oferta permanente de un tema tan importante y en la palestra de las políticas públicas y de discusión general tanto en el país como en las esferas de los organismos internacionales.

Considerando esta inconsistencia entre la práctica y el discurso promovido por el Estado y sus instituciones, se realizó una consulta con organizaciones e instituciones que operan en el ámbito de los derechos humanos, señalando las mismas que sí es una necesidad social la creación de un grado académico en derechos humanos, aun con las relativas posibilidades de incorporación laboral anteriormente explicadas. 
En esta lógica resultan fortalecidos los Diagnósticos sobre la oferta formal y no formal, pues únicamente el $38 \%$ de las organizaciones participantes tienen personal graduado en derechos humanos, $\mathrm{y}$ en el $52 \%$ de las organizaciones, los procesos de capacitación no son sistemáticos y muchas veces responden a iniciativas personales, lo que refleja que a pesar de los esfuerzos que realizan queda mucho camino por recorrer, y un paso fundamental es que la Universidad asuma parte de la responsabilidad en la educación formal y no formal de las colectividades que trabajan por el cumplimiento de derechos humanos.

Sobre el tema en particular, los datos propiciados por las consultas con 330 estudiantes de último año de secundaria destacan la existencia de una población estudiantil potencial, pues el 42,5\% del total señalaron una propensión positiva para cursar una carrera en Promoción y Protección de Derechos Humanos, contando un 27\% con intereses reales en la misma. De estos datos también se destaca que son las mujeres las más determinadas en cursar derechos humanos.

Los y las estudiantes consideraron que las poblaciones metas de derechos humanos tienen que tener como característica condiciones de vulnerabilidad o exclusión social, y para ellos el grupo prioritario deberían ser los niños, niñas y adolescentes, así como adultos mayores e indígenas.

En cuanto a la construcción de perfiles curriculares, se evidencia la necesidad de contar con un solidez en el ámbito jurídico, pues desde allí la sociedad continúa visualizando derechos humanos, sin embargo se destaca el carácter multidisciplinario de la nueva carrera, y las destrezas profesionales y las habilidades sociales propuestas por el estudiantado.

\section{Referencias bibliográficas}

Abramovich, V. (2005). Líneas de trabajo en derechos económicos, sociales y culturales: herramientas y aliados. Sur. Revista Internacional de Direitos Humanos, 2 (2). Disponible: http://www.scielo.br/scielo.php?script=sci_arttext\&pid=S1806-64452005000100009\&lng=es\&tlng=es.10.1590/ S1806-64452005000100009

Asamblea General de Naciones Unidas. (1996). Cuestiones relativas a los derechos humanos: cuestiones relativas a los derechos humanos, incluidos distintos criterios para mejorar el goce efectivo de los derechos humanos y las libertades fundamentales. Nota del Secretario General. Adición. A/51/506/Add.1.

Asamblea General de Naciones Unidas. (1996). Resolución Aprobada por la Asamblea General, 50/177. Decenio de las Naciones Unidas para la educación en la esfera de los derechos humanos. A/RES/50/177. 
Asamblea General de Naciones Unidas. (2000). Decenio de las Naciones Unidas para la educación en la esfera de los derechos humanos (1995-2004). Nota del Secretario General. A/55/360.

Asamblea General de Naciones Unidas. (2009). Consulta sobre el objeto de la segunda etapa del Programa Mundial para la educación en derechos humanos. Informe de la Alta Comisionada de las Naciones Unidas para los Derechos Humanos. $\mathrm{A} / \mathrm{HRC} / 12 / 36$.

Asamblea General de Naciones Unidas. (2010). Evaluación final de la aplicación de la primera etapa del Programa Mundial para la educación en derechos humanos. A/65/322.

Asamblea General de Naciones Unidas. (2010). Proyecto de plan de acción para la segunda etapa (2010-2014) del Programa Mundial para la educación en derechos humanos. Nota de la Alta Comisionada de las Naciones Unidas para los Derechos Humanos. A/HRC/15/28.

Asamblea General de Naciones Unidas. (2012). Informe sobre los progresos realizados en la aplicación del Programa Mundial para la educación en derechos Humanos. Informe de la Alta Comisionada de las Naciones Unidas para los Derechos Humanos A/HRC/21/20.

Blas, A. (2011). Entrevista personal. Oficina de Educación Permanente de la Universidad Nacional de Costa Rica. Setiembre de 2011.

Comité de derechos económicos, sociales y culturales. (1999). Observación general No. 13. El derecho a la educación (artículo 13 del Pacto). E/C.12/1999/10. Disponible: http://daccess-dds-ny.un.org/doc/UNDOC/GEN/G99/462/19/PDF/ G9946219.pdf?OpenElement

Comité de derechos económicos, sociales y culturales. (1999). Observación general No. 11. Planes de acción para la enseñanza primaria (artículo 14 del Pacto Internacional de Derechos Económicos, Sociales y Culturales). E/C.12/1999/4. Disponible: http://daccess-dds-ny.un.org/doc/UNDOC/GEN/G99/422/79/PDF/ G9942279.pdf?OpenElement

CONARE-OPES. (2007). Dictamen sobre la propuesta de creación de la Maestría en Derechos Humanos de la Niñez y la Adolescencia de la Universidad de Costa Rica. San José.

Consejo Económico y Social. (2001). Promoción y protección de los derechos humanos: Información y educación. Ejecución del Plan de Acción del Decenio de las Naciones Unidas para la educación en la esfera de los derechos humanos, 1995-2004. Informe de la Alta Comisionada. E/CN.4/2002/104.

Consejo Económico y Social. (2004). Promoción y protección de los derechos humanos: Información y educación. Decenio de las Naciones Unidas para la educación en la esfera de los derechos humanos (1995-2004): Informe sobre los logros y los fallos registrados en el Decenio y sobre las futuras actividades de las $\mathrm{Na}$ ciones Unidas en esta esfera. Informe del Alto Comisionado. E/CN.4/2004/93.

Consejo Económico y Social. (2004). Promoción y protección de los derechos humanos: Información y educación. Medidas complementarias del Decenio de las Naciones Unidas para la educación en la esfera de los derechos humanos (1995-2004), incluida la proclamación del Programa Mundial para la educación en derechos humanos. Informe de la Alta Comisionada. E/CN.4/2005/98. 
Cotino, L. (2012). El derecho a la educación como derecho fundamental. Especial atención a su dimensión social prestacional. Cuadernos y Debates, 221. Centro de Estudios Políticos y Constitucionales: Madrid.

González, M. (2011). Entrevista personal. Vicerrectoría de Acción Social de la Universidad de Costa Rica. Noviembre de 2011.

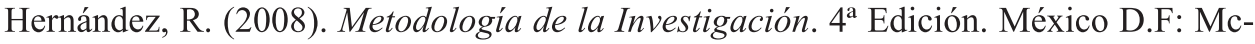
Graw Hill.

Instituto Interamericano de Derechos Humanos. (2002). Informe Interamericano de la Educación en derechos humanos. Un estudio en 19 Países. Parte I: Desarrollo Normativo. San José: Instituto Interamericano de Derechos Humanos.

Instituto Interamericano de Derechos Humanos. (2011). XXIX Curso Interdisciplinario en Derechos Humanos. Recuperado de www.iidh.ed.cr

Instituto Tecnológico de Costa Rica. (2011). Capacitese con nosotros. Recuperado de http://www.tec.ac.cr/Lists/Cursos\%20de\%20Capacitacion/AllItems.aspx

Maestría en Derechos Humanos de la Niñez y la Adolescencia. (2011). Información de la apertura de la III promoción. Correspondencia personal.

Maestría en Derechos Humanos y Educación para la Paz. (2002). Programa de Estudios. Mimeografía.

Maestría en Derechos Humanos. (s.f.). Introducción. Recuperado de http://www.uned. ac.cr/posgrados/maestrias/Derechos_Humanos/default.shtml

Maestría en Derechos Humanos. (s.f.). Requisitos de admisión. Recuperado de http:// www.uned.ac.cr/posgrados/maestrias/Derechos_Humanos/admision.shtml

Maestría en Derechos Humanos. (s.f.). Plan de Estudios. Recuperado de http://www. uned.ac.cr/posgrados/maestrias/Derechos_Humanos/plan.shtml

Maestría en Derechos Humanos. (s.f.). Características y duración. Recuperado de http://www.uned.ac.cr/posgrados/maestrias/Derechos_Humanos/caracteristicas.shtml

Maestría en Derechos Humanos. (s.f.). Costos y financiamiento. Recuperado de http:// www.uned.ac.cr/posgrados/maestrias/Derechos_Humanos/costos.shtml

Maestría profesional en derecho comunitario y derechos humanos. (2011). Información promocional para la apertura del programa. Recuperado de www.sep.ucr.ac.cr

Meoño, S. (2008). El derecho a la educación en Costa Rica. San José: Defensoría de los Habitantes de la República de Costa Rica.

Ministerio de Educación Pública (MEP). (2010). Informe de Costa Rica sobre educación en Derechos Humanos. Dirección de Promoción y Protección de Derechos Estudiantiles.

Oficina del Alto Comisionado de las Naciones Unidas para los Derechos Humanos (OACNUDH) y UNESCO. (2006). Plan de acción Programa Mundial para la educación en derechos humanos. Primera etapa. Nueva York y Ginebra.

Programa Cátedra Latinoamericana de Criminología de Derechos Humanos Alessadro Baratta. (2011). Recuperado de http://www.una.ac.cr/cab/index. php?option $=$ com_content\&view $=$ article\&id $=49 \&$ Itemid $=57$

Programa Estado de la Nación. (2011). Tercer informe del Estado de la Educación. San José: Programa Estado de la Nación. 
Universidad Nacional de Educación a Distancia. (s.f.). Proyecto de Atención a Poblaciones Vulnerables. Recuperado de http://www.uned.ac.cr/extension/atencionpoblacionesbulnerables.shtml

Universidad Nacional de Educación a Distancia. (s.f.). Proyecto Niñez y Adolescencia. Recuperado de http://www.uned.ac.cr/extension/niniesyadolecencia.shtml

Universidad para la Paz. (2011). International Law and Human Rights. Recuperado de http://www.upeace.org/academic/masters/ILHR.cfm

Universidad para la Paz. (2011). International Peace Studies. Recuperado de http:// www.upeace.org/academic/masters/IPS.cfm

Universidad para la Paz. (2011). Peace Education. Recuperado http://www.upeace.org/ academic/masters/PE.cfm

Universidad para la Paz. (2011d). Tuition Fees. Recuperado de http://www.upeace.org/ admissions/tuition.cfm

UniversidadTécnicaNacional.(2011).Programastécnicosydeacciónsocial.Recuperadodehttp:// extension.utn.ac.cr/index.php?option $=$ com content\&view $=$ article\&id $=5 \& I t e m i d=4$ 